\title{
PONTRJAGIN CLASSES OF PL SHEAVES
}

\author{
BY HOWARD OSBORN
}

\author{
Communicated by S. S. Chern, May 12, 1972
}

\begin{abstract}
Over the category of PL manifolds there is a fibered category whose objects are certain equivalence classes $[\mathscr{F}]$ of "PL sheaves" $\mathscr{F}$, to which one assigns real characteristic classes as in [2] and [3]. In particular each PL manifold $M$ possesses a distinguished (co)tangent object $[\mathscr{E}(M)]$ and a real Pontrjagin class $p([\mathscr{E}(M)])$. In this note we show that $p([\mathscr{E}(M)])$ is the image under $H^{4 *}(M ; Q) \rightarrow H^{4 *}(M ; R)$ of the Thom-Pontrjagin class of $M$.
\end{abstract}

The construction of [2] and [3] assigns total Chern classes $c([\mathscr{F}]) \in H^{2 *}(M ; R)$ to cosets $[\mathscr{F}]$ of complex PL sheaves $\mathscr{F}$ over a PL manifold $M$, and this assignment satisfies certain axioms. As in the classical case one defines the total Pontrjagin class $p([\mathscr{F}]) \in H^{4 *}(M ; R)$ of a coset $[\mathscr{F}]$ of real PL sheaves via complexification of $[\mathscr{F}]$, and here are the corresponding axioms:

if $[\mathscr{F}]$ is a coset of real PL sheaves of "rank" $m$ on a PL

$\left(\mathrm{P}_{1}\right) \quad$ manifold $M$ then the total Pontrjagin class $p([\mathscr{F}])$ is an element $1+p_{1}([\mathscr{F}])+\cdots+p_{[m / 2]}([\mathscr{F}])$ of $H^{*}(M ; R)$ with $p_{i}([\mathscr{F}]) \in H^{4 i}(M ; R)$;

$$
p\left(\Xi^{\prime}[\mathscr{F}]\right)=\Xi^{*} p([\mathscr{F}]) \in H^{4 *}(N ; R) \text { for any PL map }
$$

$$
\Xi: N \rightarrow M
$$

$$
p([\mathscr{F}] \oplus[\mathscr{G}])=p([\mathscr{F}]) \cup p([\mathscr{G}]) \text { for any cosets }[\mathscr{F}] \text { and }
$$
$[\mathscr{G}]$ over $M$;

if $[\mathscr{F}]$ contains a bona fide real vector bundle $\xi$ over $M$ (as $\left(\mathrm{P}_{4}\right) \quad$ in $[\mathbf{2}]$ or $\left.[\mathbf{3}]\right)$ then $p([\mathscr{F}])$ is the classical total Pontrjagin class $p(\xi) \in H^{4 *}(M ; R)$.

LEMMA 1. If a PL manifold $M$ happens to admit a smooth structure with tangent bundle $\tau_{M}$ then $p([\mathscr{E}(M)])=p\left(\tau_{M}\right)$.

Proof. One easily verifies as in [2] that $[\mathscr{E}(M)]$ contains $\tau_{M}$; hence it suffices to apply $\left(\mathrm{P}_{4}\right)$.

As in the smooth case one uses axioms $\left(\mathrm{P}_{1}\right),\left(\mathrm{P}_{2}\right),\left(\mathrm{P}_{3}\right)$ and the multiplicative sequence corresponding to $z^{1 / 2} /\left(\tanh z^{1 / 2}\right)$ to construct the Hirzebruch polynomial $l([\mathscr{F}]) \in H^{4 *}(M ; R)$ of the Pontrjagin class $p([\mathscr{F}])$,

AMS (MOS) subject classifications (1970). Primary 57C50, 57D20; Secondary 57C99, 57D55.

Key words and phrases. PL sheaves, Pontrjagin classes, PL cobordism, Hirzebruch index formula, Thom construction. 
and one defines the $L$-genus $L(M)$ of any compact oriented PL manifold $M$ of dimension $4 n$ by setting $L(M)=\left\langle l_{n}([\mathscr{E}(M)]),[M]\right\rangle$ for the fundamental class $[M] \in H_{4 n}(M ; R)$. The index $I(M)$ is defined as usual, and the classical Hirzebruch index formula combines with Lemma 1 to guarantee that $L(M)=I(M)$ whenever the PL manifold $M$ happens to have a smooth structure.

Lemma 2. $L(M)=I(M)$ for any compact oriented PL manifold $M$ of dimension $4 n$.

Proof. One easily verifies as in the smooth case that

$$
L(M+N)=L(M)+L(N), L(-M)=-L(M), L(M \times N)=L(M) \cdot L(N)
$$

and $L$ (boundary) $=0$, so that $L$ may be regarded as a homomorphism $\Omega_{*}^{\mathrm{PL}} \otimes R \rightarrow R$. Hence it will suffice to verify that the homomorphism $L$ agrees on generators with the corresponding homomorphism $I: \Omega_{*}^{\mathrm{PL}} \otimes R \rightarrow R$. But the homomorphism $\Omega_{*} \rightarrow \Omega_{*}^{\mathrm{PL}}$ is injective (by $\left(\mathrm{P}_{4}\right)$, for example), which yields an exact sequence

$$
0 \rightarrow \Omega_{q} \rightarrow \Omega_{q}^{\mathrm{PL}} \rightarrow \Omega_{q}^{\mathrm{PL}} / \mathbf{\Omega}_{q} \rightarrow 0
$$

in each dimension $q$, and Williamson showed in [5] that each $\Omega_{q}^{\mathrm{PL}} / \Omega_{q}$ is finite. Hence $\Omega_{*} \otimes R \rightarrow \Omega_{*}^{\mathrm{PL}} \otimes R$ is an isomorphism, so that each class in $\Omega_{*}^{\mathrm{PL}} \otimes R$ contains at least one smooth manifold; but we already know from Lemma 1 that $L(M)=I(M)$ for smooth manifolds $M$.

Now for any PL manifold $M$ let $P(M) \in H^{4 *}(M ; R)$ be the image under $H^{*}(M ; Q) \rightarrow H^{*}(M ; R)$ of the rational Pontrjagin class constructed by Thom in [4]. (See [1] for an alternate version of Thom's construction.) Thom's construction established the existence of unique rational classes satisfying certain axioms, which we translate as follows into real cohomo$\log$ :

for each oriented PL manifold $M$ of dimension $m$ the class

( $\left.\mathrm{T}_{1}\right) \quad P(M)$ is of the form $1+P_{1}(M)+\cdots+P_{[m / 2]}(M)$ with $P_{i}(M) \in H^{4 i}(M ; R)$;

to each embedding $i: N \rightarrow M$ of one oriented PL manifold

$\left(\mathrm{T}_{2}\right)$ into another one can assign a "normal" class $Q(N) \in H^{4 *}(N ; R)$ satisfying $P(N) \cup Q(N)=i^{*} P(M)$;

if $l^{\prime}(M) \in H^{4 *}(M ; R)$ is the Hirzebruch polynomial constructed from $z^{1 / 2} /\left(\tanh z^{1 / 2}\right)$ and $P(M)$ then the $L$-genus defined for any $4 n$-dimensional compact oriented PL manifold $M$ by $L^{\prime}(M)=\left\langle l_{n}^{\prime}(M),[M]\right\rangle$ satisfies $L^{\prime}(M)=I(M)$.

Here is the main result of this note, which permits one to conclude 
that the Pontrjagin classes $p([\mathscr{F}])$ form an extension of the ThomPontrjagin construction to a reasonable fibered category over the category of PL manifolds:

Proposition. $p([\mathscr{E}(M)])=P(M) \in H^{4 *}(M ; R)$ for any $P L$ manifold $M$.

Proof. It suffices to verify that the classes $p([\mathscr{E}(M)])$ satisfy Thom's axioms. But $\left(\mathrm{T}_{1}\right)$ is an immediate consequence of $\left(\mathrm{P}_{1}\right),\left(\mathrm{T}_{2}\right)$ follows easily from $\left(\mathrm{P}_{2}\right)$ and $\left(\mathrm{P}_{3}\right)$, and $\left(\mathrm{T}_{3}\right)$ follows from Lemma 2 .

\section{REFERENCES}

1. J. Milnor, Lectures on characteristic classes, Mimeographed notes, Princeton University, Princeton, N.J., 1958.

2. H. Osborn, Differential geometry in PL, Mimeographed notes, University of Illinois, Urbana-Champaign, Ill., 1972.

3.,$- P L$ sheaves and their characteristic classes Bull. Amer. Math. Soc. 78 (1972), 787-791.

4. $\mathrm{R}$. Thom, Les classes caractéristiques de Pontrjagin des variétés triangulées, Internat. Sympos. on Algebraic Topology, Universidad Nacional Autónoma de México and UNESCO, Mexico City, 1958, pp. 54-67. MR 21 \#866.

5. R. E. Williamson, Cobordism of combinatorial manifolds, Ann. of Math. (2) 83 (1966), 1-33. MR 32 \# 1715.

Department of Mathematics, University of Illinois at Urbana-Champaign, URBANA, ILLINOIS 61801 (current address)

Department of Mathematics, Stanford University, Stanford, California 94305 (Current address from February 1973-August 1973.) 